\title{
Variables separation of the spectral BRDF for better understanding color variation in special effect pigment coatings
}

\author{
Alejandro Ferrero, * Ana María Rabal, Joaquín Campos, Alicia Pons, and María Luisa Hernanz \\ Instituto de Óptica, Consejo Superior de Investigaciones Científicas (CSIC), Madrid 28006, Spain \\ ${ }^{*}$ Corresponding author: alejandro.ferrero@csic.es
}

Received November 2, 2011; revised January 25, 2012; accepted February 21, 2012;

posted February 24, 2012 (Doc. ID 157601); published May 11, 2012

\begin{abstract}
A type of representation of the spectral bidirectional reflectance distribution function (BRDF) is proposed that distinctly separates the spectral variable (wavelength) from the geometrical variables (spherical coordinates of the irradiation and viewing directions). Principal components analysis (PCA) is used in order to decompose the spectral BRDF in decorrelated spectral components, and the weight that they have at every geometrical configuration of irradiation/viewing is established. This method was applied to the spectral BRDF measurement of a special effect pigment sample, and four principal components with relevant variance were identified. These four components are enough to reproduce the great diversity of spectral reflectances observed at different geometrical configurations. Since this representation is able to separate spectral and geometrical variables, it facilitates the interpretation of the color variation of special effect pigments coatings versus the geometrical configuration of irradiation/viewing. (c) 2012 Optical Society of America
\end{abstract}

OCIS codes: $\quad 330.1710,330.1720,300.6550,290.1483$

\section{INTRODUCTION}

Coatings with special effect pigments [1] can strongly change color with the irradiation/viewing geometry-in lightness, in chromaticity, and, unlike the metallic coatings, in hue. This ability to change color makes them very attractive for the industry, meeting such diverse applications as automotive coatings, cosmetics, or currency (anticounterfeit) [2-7].

This kind of coating consists of a transparent substrate with embedded flakes of special effect pigments, which are horizontally arranged and produce interference due to their layered structure. The flakes are normally irregularly shaped, with a diameter between 5 and $50 \mu \mathrm{m}$. Since they tend to be parallel to the coating surface, the reflectance is higher towards the specular direction. They are usually modeled as three different layers: a clear coat, an intermediate region where the pigments are located, and a base coat. The multicolor effect is caused by light interference on this layered structure $[\underline{3}, 8,9]$. The specular component is mainly produced in the clear coat, while the diffuse reflection is mainly produced in the base coat and the hue variation is mainly produced in the intermediate region, which reflects part of the light in a specular way.

The increasingly popularity of these coatings demands the development of new techniques and instruments to characterize them [3,10-18], that is, to determine the relation between the color variables of the coating (spectral distribution, hue, or chroma) and the different irradiation/viewing geometrical configurations. The global solution of this problem lies in the complete knowledge of the spectral bidirectional reflectance distribution function (BRDF) of these coatings, because this distribution function allows the complete spectral reflectance to be calculated, and, therefore the color for any geometrical configuration (irradiation and viewing directions and solid angles) and spectral distribution of the irradiation [19]. Nowadays, the spectral BRDF has to be measured, requiring complex and fully automated instruments for a wide range of irradiation/viewing geometries, which are only affordable for few well-equipped laboratories. In addition, proper conceptual frames have also to be developed in order to represent and understand the multiangle measurements.

A type of representation of the spectral BRDF is proposed that distinctly separates the spectral variable (wavelength) from the geometrical variables (spherical coordinates of the irradiation and viewing directions). Principal components analysis (PCA) is used in order to decompose the spectral BRDF in decorrelated spectral components. This representation facilitates the interpretation of the color variation of special effect pigments coatings versus the irradiation/viewing geometry.

The measurement and representation of the BRDF of the special effect pigment material Colorstream T20-02 WNT Arctic Fire is discussed in the paper. This material is based on synthetically manufactured silicon dioxide platelets coated with titanium dioxide. This pigment shows a color shift from subtle turquoise through brilliant silver to metallic red hues [20].

The measurement of the BRDF was carried out by the gonio-spectrophotometer GEFE [21-23], developed in the Spanish Council for Scientific Research's Optics Institute (IO-CSIC). It consists of a fixed, uniform, and collimated light source and a robot-arm that places the sample, making it possible to change automatically and simultaneously the irradiation direction (defined with the spherical coordinates $\theta_{i}$ and $\left.\phi_{i}\right)$ and the viewing one $\left(\theta_{s}, \phi_{s}\right)$. These spherical coordinates are defined relative to the sample coordinate system, whose 


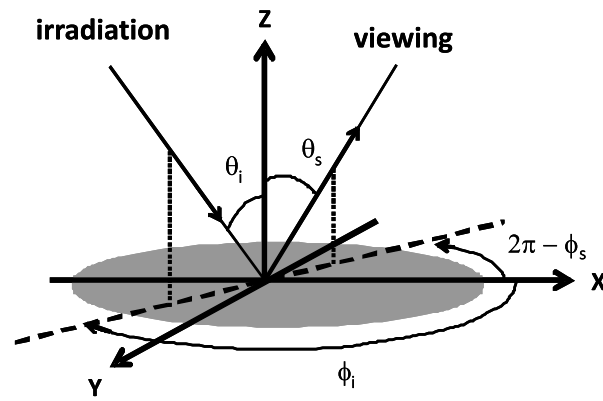

Fig. 1. Sample coordinate system showing spherical coordinates for irradiation $\left(\theta_{i}, \phi_{i}\right)$ and viewing directions $\left(\theta_{s}, \phi_{s}\right)$.

$z$ axis is parallel to the sample's normal direction (Fig. 1). It is remarkable that, thanks to its periscopic system with a beamsplitter, it is possible to measure under actual retro-reflection conditions. For the data acquisition, a CS-2000 Konica Minolta spectroradiometer is used. This device operates within the visible range (380 $\mathrm{nm}-780 \mathrm{~nm}$ ), performing spectral measurements with a $1 \mathrm{~nm}$ spectral sampling interval and a $4 \mathrm{~nm}$ bandwidth.

The BRDF was sampled at 400 different angular configurations; every one of them corresponds to a specific combination of $\theta_{i}$ and $\theta_{s}$ (which take the values $0^{\circ}, 10^{\circ}, 20^{\circ}, 30^{\circ}, 40^{\circ}$, $50^{\circ}, 60^{\circ}$, and $70^{\circ}$ ), $\phi_{i}$ (which takes only the value $0^{\circ}$ ) and $\phi_{s}$ (which takes the values $0^{\circ}, 30^{\circ}, 60^{\circ}, 90^{\circ}, 120^{\circ}, 150^{\circ}$, and $180^{\circ}$ ).

\section{FORMALISM FOR THE SEPARATION OF VARIABLES OF THE SPECTRAL BRDF}

Color of an object is determined by the optical radiation it reflects, which can be determined in each geometry from the pertinent integration of the spectral BRDF. But the spectral BRDF is a complex function because it involves a great amount of data, which makes tedious this method to obtain color. In order to simplify this problem, spectral BRDF can be obtained from a linear combination of a short number of spectral distributions with weighting coefficients dependant on the irradiation/viewing geometry [24]. These spectral distributions can be obtained with different boundary conditions. In this paper, we propose to express the spectral BRDF as a sum of components where spectral and geometrical variables are separated in this way:

$$
\begin{aligned}
f_{r}\left(\theta_{i}, \phi_{i} ; \theta_{s}, \phi_{s} ; \lambda\right)= & \left\langle f_{r}\left(\theta_{i}, \phi_{i} ; \theta_{s}, \phi_{s}\right)\right\rangle_{\lambda}[1 \\
& \left.+\sum_{j=1}^{M} c_{j}\left(\theta_{i}, \phi_{i} ; \theta_{s}, \phi_{s}\right) H_{j}(\lambda)\right] .
\end{aligned}
$$

This equation corresponds to the multiplication of two factors: the spectral average of $f_{r}\left(\left\langle f_{r}\right\rangle_{\lambda}\right)$ with just geometrical dependence, and the sum of $M+1$ addends or components (within square brackets), the first one being the unity. Every addend is factorized in two: one containing the spectral information, $H_{j}(\lambda)$ (with an average value of 0 and a standard deviation value of 1 ), and another factor containing the geometrical information, $c_{j}\left(\theta_{i}, \phi_{i} ; \theta_{s}, \phi_{s}\right)$, that can be regarded as the weight of every spectral distribution at the different geometrical configuration.

Written this way, the calculations required to obtain the spectral reflectance or spectral reflectance factors are simpli- fied, because it is not necessary to integrate the spectral distributions, but only their weights.

Experimental data of spectral BRDF can be arranged as in Eq. (1) by the application of the multivariate technique of PCA [24-27]. This analysis tool allows any BRDF spectrum at any geometrical configuration to be expressed as a linear combination of a few spectra [eigenspectra, $A_{j}(\lambda)$ ], which can be related to $H_{j}(\lambda)$. The weights $c_{j}\left(\theta_{i}, \phi_{i} ; \theta_{s}, \phi_{s}\right)$ in Eq. (1) can be related to the eigenvectors $e_{j}\left(\theta_{i}, \phi_{i} ; \theta_{s}, \phi_{s}\right)$ obtained from the PCA. The main drawback of this type of analysis is that the eigenspectra have no physical meaning because they are obtained from a purely statistical analysis of a spectral distributions set forming the spectral BRDF of the sample, so that they cannot be directly related to sample's physical parameters that govern the phenomena of light reflection on it.

In this work, these eigenspectra and eigenvectors were calculated using the PCA as explained in [24] on the relative experimental data:

$$
f_{r, \text { rel }}\left(\theta_{i}, \phi_{i} ; \theta_{s}, \phi_{s} ; \lambda\right)=\frac{f_{r}\left(\theta_{i}, \phi_{i} ; \theta_{s}, \phi_{s} ; \lambda\right)}{\left\langle f_{r}\left(\theta_{i}, \phi_{i} ; \theta_{s}, \phi_{s}\right)\right\rangle_{\lambda}}-1
$$

To obtain a $H_{j}(\lambda)$ with standard deviation of value 1 , the relation between $A_{j}(\lambda)$ and $H_{j}(\lambda)$ can be written as

$$
H_{j}(\lambda)=\frac{A_{j}(\lambda)}{\sigma\left[A_{j}(\lambda)\right]},
$$

where $\sigma\left[A_{j}(\lambda)\right]$ is the standard deviation of $A_{j}(\lambda)$.

Thus, the relation between the eigenvector $e_{j}\left(\theta_{i}, \phi_{i} ; \theta_{s}, \phi_{s}\right)$ and $c_{j}\left(\theta_{i}, \phi_{i} ; \theta_{s}, \phi_{s}\right)$ has to be rewritten as

$$
c_{j}\left(\theta_{i}, \phi_{i} ; \theta_{s}, \phi_{s}\right)=e_{j}\left(\theta_{i}, \phi_{i} ; \theta_{s}, \phi_{s}\right) \sigma\left[A_{j}(\lambda)\right]
$$

Finally, to facilitate the chromatic interpretation of the results, we used the criterion $\sum_{j=1}^{M} c_{j}(\lambda) \geq 0$; that is, both $c_{j}$ and $H_{j}$ will be multiplied by -1 if $\sum_{j=1}^{M} e_{j}(\lambda)$ is lower than 0 .

\section{RESULTS}

The values of $\left\langle f_{\mathrm{r}}\left(\theta_{i}, \phi_{i} ; \theta_{s}, \phi_{s}\right)\right\rangle_{\lambda}$ calculated from the measurements are shown in Fig. 2, where every plot corresponds to a different $\theta_{i}$. The half-plane containing the incident direction $\left(\phi_{s}=0^{\circ}\right)$ corresponds to negative $\theta_{s}$, and the half-plane containing the specular direction $\left(\phi_{s}=180^{\circ}\right)$ corresponds to positive $\theta_{s}$. For the sake of the comparison, the ideal constant value $1 / \pi$, i.e., the $\mathrm{BRDF}$ for the perfectly reflecting diffuser (PRD), is also plotted. In the case of the out-of-plane representation, the half-planes $30^{\circ}$ apart from the half-planes containing incident direction (negative $\theta_{\mathrm{s}}, \phi_{s}=30^{\circ}$ ) and specular reflection (positive $\theta_{s}, \phi_{s}=150^{\circ}$ ) are represented.

A maximum value around the specular direction is observed at every $\theta_{i}$. This specular reflection of the BRDF is less sharp and decreases as viewing polar angles increase.

The four relevant components $H_{j}(\lambda)$ containing the $99.4 \%$ of the total relative variance of the data are shown in Fig. $\underline{3}$, arranged from the highest to the lowest variance. The remaining $0.6 \%$ of the variance does not contain relevant spectral information; therefore, the BRDF was expressed as in Eq. (1) using only these four components. 

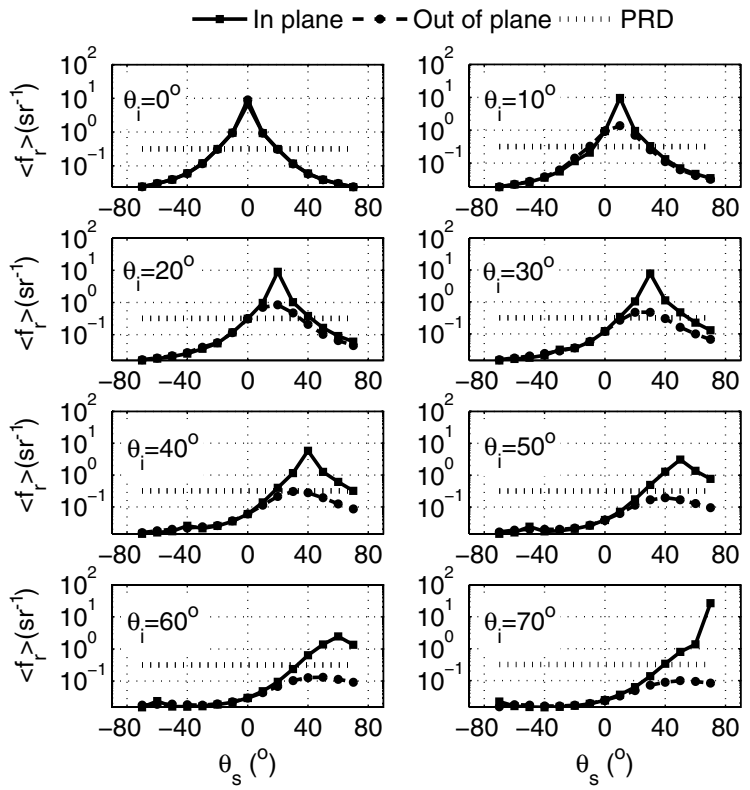

Fig. 2. Representation of $\left\langle f_{r}\left(\theta_{i}, \phi_{i} ; \theta_{s}, \phi_{s}\right)\right\rangle_{\lambda}$ versus viewing polar angles. Every plot corresponds to a different incident polar angle $\left(\theta_{i}\right)$.

$H_{1}(\lambda)$ has an absolute maximum at $490 \mathrm{~nm}$, a relative minimum at $590 \mathrm{~nm}$, and a relative maximum at $660 \mathrm{~nm}$, and it strongly decays below $430 \mathrm{~nm} . H_{2}(\lambda)$ has a relative maximum at $500 \mathrm{~nm}$ and two relative minima near $420 \mathrm{~nm}$ and $600 \mathrm{~nm}$, respectively, and increases toward longer wavelengths, producing reddening when increasing the weight $c_{2} . H_{3}(\lambda)$ has an absolute maximum at $620 \mathrm{~nm}$, a relative maximum of half height at $445 \mathrm{~nm}$, and a minimum at $520 \mathrm{~nm}$. Finally, $H_{4}(\lambda)$ has its absolute maximum at $550 \mathrm{~nm}$, a relative maximum at $410 \mathrm{~nm}$, and a minimum at $450 \mathrm{~nm}$.

The corresponding weights $c_{j}$ at different geometrical configurations are represented in Figs. $\underline{4}$ (in-plane) and $\underline{5}$ (out-of-plane).

In Fig. 4, it is observed that the weights $c_{i}$ have a different behavior in the half-plane containing the incident direction
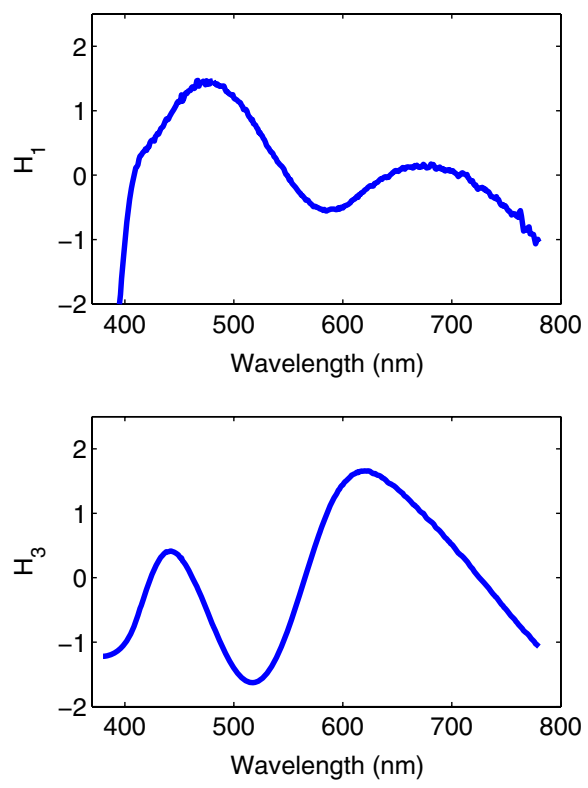
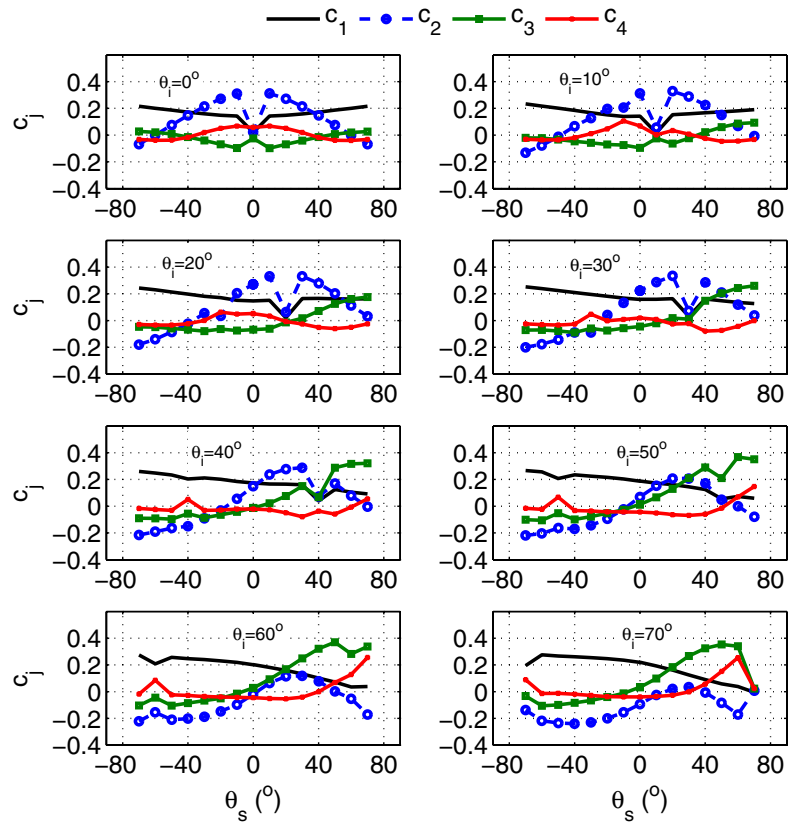

Fig. 4. (Color online) Weights $c_{j}$ at different in-plane geometrical configurations corresponding to $\mathrm{H}_{1}, \mathrm{H}_{2}, \mathrm{H}_{3}$, and $\mathrm{H}_{4}$, respectively.

(negative $\theta_{s}$ ) than in the half-plane containing the specular direction (positive $\theta_{s}$ ). In the first half-plane, the behavior is more homogeneous: $c_{1}$ is the dominant weight and $c_{3}$ and $c_{4}$ do not change too much. The trend of $c_{2}$ changes much more with $\theta_{i}$. In addition, for $\theta_{i}<40^{\circ}$, its value can be even higher than the value of $c_{1}$, whereas, for higher $\theta_{i}$, its value is comparable to $c_{3}$ and $c_{4}$. On the other hand, in the other halfplane (positive $\theta_{s}$ ), the relative behavior of these weights is different: $c_{1}$ and $c_{2}$ decrease with $\theta_{i}$, whereas $c_{3}$ and $c_{4}$ increase. $c_{1}$ is the weightless variable versus the viewing angle, decreasing monotonically when this angle increases, except in the minima appearing at specular angles. On the other hand, relative minima and maxima for some weights are observed both at specular and retro-reflection configurations.
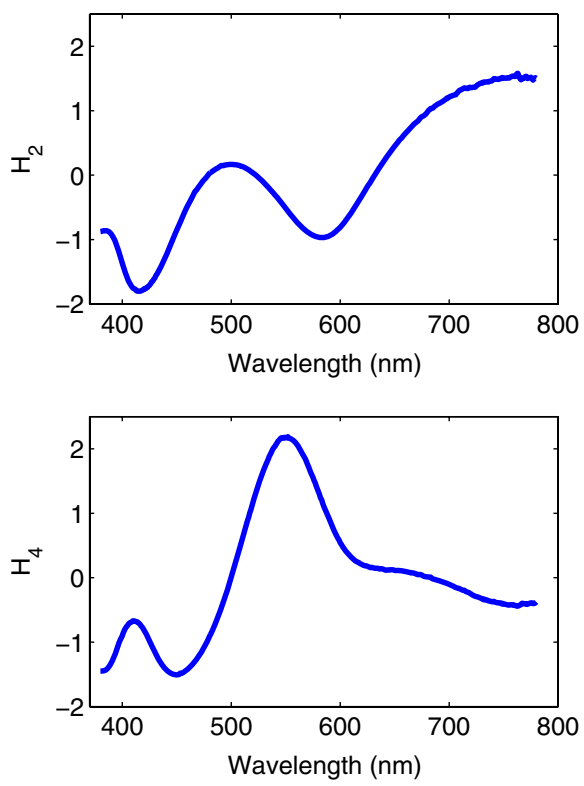

Fig. 3. (Color online) The four components of $H_{j}(\lambda)$ containing $99.4 \%$ of the total relative variance of the data, arranged from the highest to the lowest variance. 

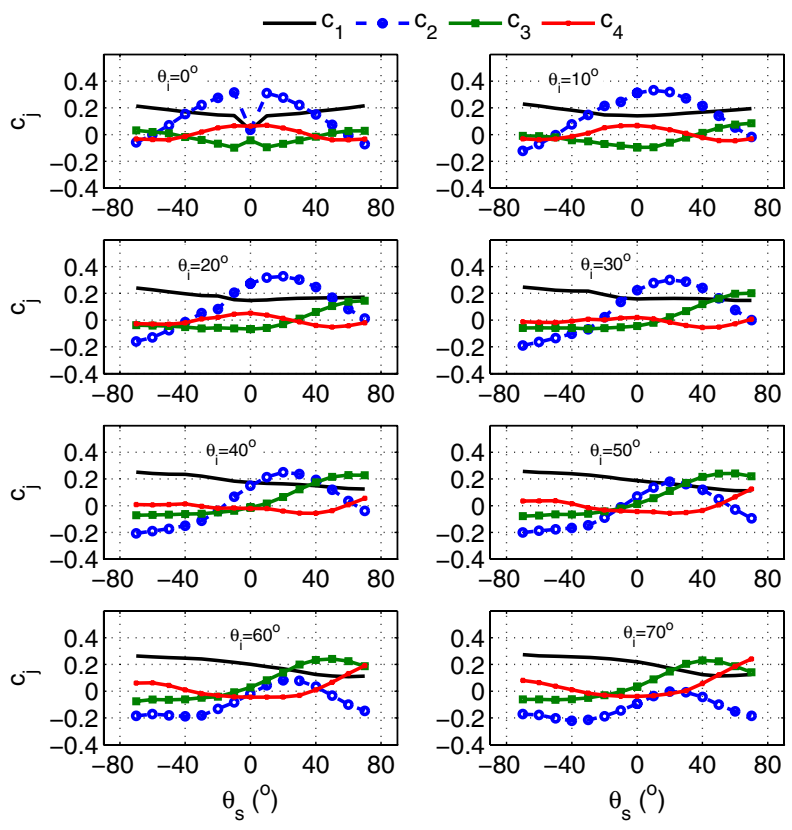

Fig. 5. (Color online) Weights $c_{j}$ at different out-of-plane geometrical configurations corresponding to $\mathrm{H}_{1}, \mathrm{H}_{2}, \mathrm{H}_{3}$, and $\mathrm{H}_{4}$, respectively.

A similar structure to the one in Fig. 4 (in-plane weights) is observed in the representation of the out-of-plane weights (Fig. 5). The only difference is that, except for $\theta_{i}=0^{\circ}$, no retro-reflection and specular features appear.

\section{DISCUSSION}

The PCA-separation of the spectral variables from the geometrical variables simplifies many calculations. For instance, the calculation of the spectral reflectance or the spectral radiance factor, which usually requires a different double integration in spherical coordinates for every wavelength [19], is simplified because the number of integrations is reduced to the number of weights $c_{j}$, four in this case. Again, the radiance factor or the reflectance would be represented as a linear combination of the components $H_{j}$ with weights $c_{j}$.

In order to better understand the visual appearance of the sample, we integrated to calculate the spectral hemisphericaldirectional (HD) radiance factor from the PCA result shown in previous figures. This spectral radiance factor refers to the radiance in a given direction when the surface is illuminated from all directions, which is an approximation of a very common case (scattered sunlight irradiation).

Integration-calculated weights $C_{H D j}$ corresponding to the $\mathrm{HD}$ radiance factor are represented in Fig. $\underline{6}$ as a function of the viewing direction.

It is observed that the most relevant contribution to the reflectance factor comes from $H_{1}$ at any viewing angle. The contribution of $\mathrm{H}_{2}$ is higher than those of $\mathrm{H}_{3}$ and $\mathrm{H}_{4}$ for viewing angles up to around $\theta_{s}=30^{\circ}$. This situation is reversed for values of $\theta_{s}$ higher than approximately $50^{\circ}$. There is an intermediate position (around $40^{\circ}$ ) where the contribution of $H_{1}$ relative to $\mathrm{H}_{2}$ and $\mathrm{H}_{3}$ is maximum. The changing weights of the components are responsible for the change in the perceived hue at different geometrical configurations, since the resultant spectra are always a mixture of components without a constant relation.

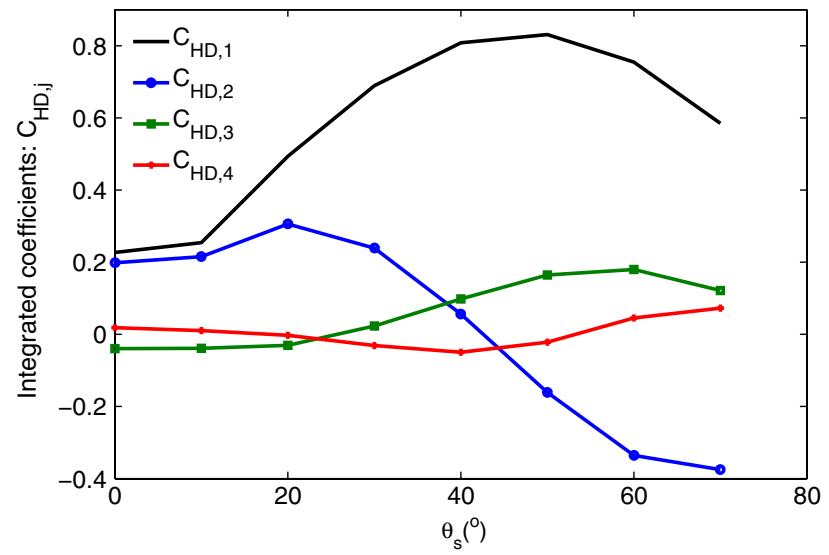

Fig. 6. (Color online) Integration-calculated weights $C_{H D, j}$ corresponding to the HD radiance factor.

Reciprocally, a constant hue will be observed when the relation between the weights is constant. Such a situation occurs around some configurations when the observer is located in the half-plane containing the irradiation direction (see Figs. $\underline{4}$ and 5). This result may be explained by the almost parallel alignment of the platelet pigments in the special effect coating (whose presence produces an important part of the change of hue), because the light is specularly reflected by them.

The prevalence of $H_{1}$, particularly for great viewing angles, is an interesting result. Considering that the weight coefficient of $H_{1}$ does not change so much with the viewing angle (see Figs. 4 and 5 ), it can be said that $H_{1}$ could be greatly influenced by the reflectance of base coating.

$\mathrm{H}_{4}$ is the component that contributes the least to the reflectance (Fig. 6), and its weight varies slightly with the viewing angle. Furthermore, as shown in Figs. 4 and 5, its contribution to the BRDF has a certain importance in the directions close to specular reflection. Therefore, $H_{4}$ could be further influenced by the reflection on the clear coat.

$\mathrm{H}_{2}$ and $\mathrm{H}_{3}$ may correspond with the chromatic changes observed on the sample (special appearance effect) from different directions when the sample is hemispherically illuminated, because their weight coefficients have very different significance along the range of viewing angles, resembling the effect produced by the interference pigments.

As mentioned before, $H_{1}$ may be related to the reflection on the base coat, but it is quite likely that it also represents in some extent the spectral reflection on the interference pigments and on the clear coat, because $H_{1}$ comes from a statistical analysis.

If we consider that reversed spectral characteristics (configurations where $c_{j}<0$ ) are difficult to handle to study chromatic tendencies, it is possible to modify the BRDF expression proposed in Eq. (1) in order to obtain a new base of representation for it with a new set of weight coefficients with positive values. Let us rewrite Eq. (1) in this modified way:

$$
\begin{aligned}
f_{r}= & \left\langle f_{r}\right\rangle_{\lambda}\left[1+c_{1} H_{1}^{\prime}+\left(c_{2}-c_{1} c_{2, \min }\right) H_{2}+\left(c_{3}-c_{1} c_{3, \min }\right) H_{3}\right. \\
& \left.+\left(c_{4}-c_{1} c_{4, \min }\right) H_{4}\right],
\end{aligned}
$$

where we simply added and subtracted within the square brackets the term $c_{1} \times\left(c_{2, \min } H_{2}+c_{3, \min } H_{3}+c_{4, \min } H_{4}\right)\left(c_{j, \text { min }}\right.$ 


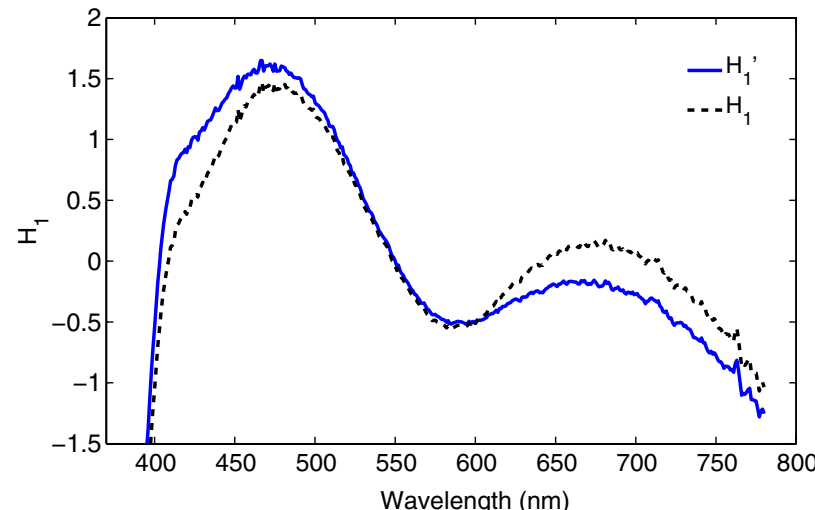

Fig. 7. (Color online) Comparison between the principal component $H_{1}$ and the modified $H_{1}\left(H_{1}^{\prime}\right)$.

is the minimum value of the ratio $c_{j} / c_{1}$ ), and denoted $H_{1}^{\prime}$ as

$$
H_{1}^{\prime}=H_{1}+c_{2, \min } H_{2}+c_{3, \min } H_{3}+c_{4, \min } H_{4} .
$$

By definition, the weights of $H_{j}$ are never negative after this modification. The new $H_{1}^{\prime}$ might be closer to the base coat reflectance of the sample since a part of the components $H_{2}, H_{3}$, and $H_{4}$ is subtracted from $H_{1}$. A comparison between $H_{1}$ and $H_{1}^{\prime}$ is shown in Fig. $\underline{7}$, where $H_{1}^{\prime}$ and $c_{1}$ were again normalized as described in Eqs. (3) and (4). $H_{1}^{\prime}$ has a higher value at short wavelengths with respect to long wavelengths than $H_{1}$, as expected, since proportions of $H_{2}$ and $H_{3}$ were subtracted, both of them being spectral distributions with higher contribution at longer wavelengths.

Finally, it should be noted that the way we defined the input spectra [Eq. (2)] favors the accurate obtaining of the components with higher relative variance, but, in some cases, it could be detrimental to the specular analysis, whose spectral distributions may have high absolute variance but very low relative variance. A solution for this problem would be to use as input spectra for the PCA:

$f_{r, \mathrm{abs}}\left(\theta_{i}, \phi_{i} ; \theta_{s}, \phi_{s} ; \lambda\right)=f_{r}\left(\theta_{i}, \phi_{i} ; \theta_{s}, \phi_{s} ; \lambda\right)-\left\langle f_{r}\left(\theta_{i}, \phi_{i} ; \theta_{s}, \phi_{s}\right)\right\rangle_{\lambda}$.

Thus, the analysis will preferably return the components related to the highest absolute variance.

\section{CONCLUSIONS}

The measurement geometry dependence of the spectral BRDF of a special effect surface is analyzed in this article. A PCAbased representation of the spectral BRDF is proposed that distinctly separates the spectral variable (wavelength) from the geometrical variables (spherical coordinates of the irradiation and viewing directions). This representation facilitates the interpretation of the color variation with the geometrical configuration of irradiation/viewing of special effect pigments coatings, and it is a simple formalism that may be of help to developing spectral models of the BRDF of these surfaces or to determining a significant set of measuring angular configurations.
Four principal components with relevant variance were identified after the PCA. The great diversity of BRDF spectra obtained at different geometrical configurations can be reproduced as a linear combination of only these four components. Therefore, these components allow the color of the surface at any irradiation/viewing geometry to be calculated. From the examination of the geometrical distribution of the weighting coefficients, it seems consistent to identify a relation between the first component and the spectral reflectance of the base coat, between the fourth component and the spectral reflectance on the surface of the clear coat, and between the second and third components and the special effect (change of hue) produced by the interference pigments.

\section{ACKNOWLEDGMENTS}

The authors are grateful to Dr. F. M. Martínez-Verdú (Department of Optics, University of Alicante) for providing us with the sample of Colorstream T20-02 WNT Arctic Fire. They are also grateful to "Plan Nacional de Física" for funding this work (FIS2010-19756-E), and to the Junta de Ampliación de Estudios (JAE) Program from Consejo Superior de Investigaciones Científicas (CSIC) for providing us with a researcher in formation.

\section{REFERENCES}

1. F. J. Maile, G. Pfaff, and P. Reynders, "Effect pigments-past, present and future" Progr. Organic Coatings 54, 150-163 (2005).

2. M. Mikula, M. Ceppan, and K. Vaško, "Gloss and goniocolorimetry of printed materials," Color Res. Appl. 28, 335-342 (2003).

3. M. E. Nadal and E. A. Early, "Color measurements for pearlescent coatings," Color Res. Appl. 29, 38-42 (2004).

4. H. J. Streitberger and K. F. Dössel, Automotive Paints and Coatings (Wiley-VCH, 2008).

5. P. Dutré, K. Bala, and P. Bekaert, Advanced Global Illumination (A K Peters, 2006).

6. J. Dorsey, H. Rushmeier, and F. Sillion, Digital Modelling of Material Appearance (Elsevier, 2007).

7. G. Müller, J. Meseth, M. Sattler, R. Sarlette, and R. Klein, "Acquisition, synthesis and rendering of bidirectional texture functions," Comput. Graph. Forum 24, 83-109 (2005).

8. G. Baba, "Gonio-spectrophotometric analysis of pearl-mica paint," Die Farbe 37, 99-110 (1990)

9. T. A. Germer and M. E. Nadal, "Modeling the appearance of special effect pigment coatings," Proc. SPIE 4447, 77-86 (2001).

10. M. E. Nadal and T. A. Germer, "Colorimetric characterization of pearlescent coatings," in Proceedings of AIC Color (2001), pp. 757-760.

11. B. Parker, "Color shift of light interference pigments," Surf. Coat. Aust. 39, 10-13 (2002).

12. F. Leloup, P. Hanselaer, M. Pointer, and J. Versluys, "Characterization of gonio-apparent colours," in Proceedings of AIC Color (2005), pp. 515-518.

13. C. A. Nicholls, Visual and instrumental characterisation of special-effect colours, Ph.D. thesis (University of Leeds, 2000).

14. R. Besold, "Metallic effect-characterization, parameter and methods for instrumentally determination," Die Farbe 37, 79-85 (1990).

15. DIN standard 6175-2, "Colour tolerances for automobile lacquer finishes, Part 2: Effect lacquer finishes” (2001).

16. DIN standard 6175-2, "Tolerances for automotive paints, Part 2: Goniochromatic paints (Farbtoleranzen für Automobillackierungen-Teil 2: Effektlackierungen)" (2001).

17. E. Chorro, E. Perales, F. M. Martínez-Verdú, J. Campos, and A. Pons, "Colorimetric and spectral evaluation of the optical anisotropy of metallic and pearlescent samples," J. Mod. Opt. 56, 1457-1465 (2009). 
18. E. Kirchner, G. J. Van den Kiebomm, L. Njo, R. Supér, and R. Gottenbos, "Observation of visual texture of metallic and pearlescent materials," Color Res. Appl. 32, 256-266 (2007).

19. F. E. Nicodemus, J. C. Richmond, J. J. Hsia, I. W. Ginsberg, and T. Limperis, Geometrical Considerations and Nomenclature for Reflectance, National Bureau of Standards Monograph 161 (National Bureau of Standards, 1977).

20. Colorstream T20-02 WNT Artic Fire, Product Information, Colorstream. Merck KgaA (2004).

21. A. M. Rabal, A. Ferrero, J. L. Fontecha, A. Pons, J. Campos, A. Corróns, and A. M. Rubiño, "Gonio-spectrophotometer for low-uncertainty measurements of bidirectional scattering distribution function (BSDF)," in Proceedings of CIE Expert Symposium on Spectral and Imaging Methods for Photometry and Radiometry, Publication CIE x036: 2010 (CIE, 2010), pp. 79-84.

22. A. M. Rabal, A. Ferrero, J. L. Fontecha, A. Pons, J. Campos, and A. Corróns, "Gonio-spectrophotometer for bidirectional scattering distribution function (BSDF) measurements," Opt. Pura Apl. 44, 137-147 (2011).
23. A. M. Rabal, A. Ferrero, J. Campos, J. L. Fontecha, A. Pons, A. M. Rubiño, and A. Corróns, "Automatic gonio-spectrophotometer for the absolute measurement of the spectral BRDF at inand out-of-plane retroreflection geometries," Metrologia 49, 213-223 (2012).

24. A. Ferrero, J. Campos, A. M. Rabal, A. Pons, M. L. Hernanz, and A. Corróns, "Principal components analysis on the spectral bidirectional reflectance distribution function of ceramic colour standards," Opt. Express 19, 19199-19211 (2011).

25. A. Ferrero, J. Alda, J. Campos, J. M. López-Alonso, and A. Pons, "Principal components analysis of the photoresponse nonuniformity of a matrix detector," Appl. Opt. 46, 9-17 (2007).

26. J. L. Simonds, "Application of characteristic vector analysis to photographic and optical response data," J. Opt. Soc. Am. 53, 968-971 (1963).

27. J. M. López-Alonso, J. Alda, and E. Bernabéu, "Principalcomponent characterization of noise for infrared images," Appl. Opt. 41, 320-331 (2002). 